\title{
Optimal sensing and control of run-and-tumble chemotaxis
}

\author{
Kento Nakamura $\left[\right.$ and Tetsuya J. Kobayashi ${ }^{*}{ }^{*}$ \\ Department of Mathematical Informatics, Graduate School of Information Science and Technology, The University of Tokyo, \\ 7-3-1, Hongo, Bunkyo-ku, Tokyo 113-8654, Japan
}

(Received 30 June 2021; accepted 10 January 2022; published 15 February 2022)

\begin{abstract}
Run-and-tumble chemotaxis is a representative search strategy for odor sources by sensing its spatial gradients. The optimal ways of sensing and control in run-and-tumble chemotaxis have been theoretically analyzed to elucidate the efficiency of the strategies implemented in organisms. However, because of theoretical difficulties, most attempts have been limited to either linear or deterministic analysis, even though real biological chemotactic systems involve considerable stochasticity and nonlinearity in their sensory processes and controlled responses. In this study, by combining the theories of optimal filtering and Kullback-Leibler control of a partially observed Markov decision process (POMDP), we derive an optimal and fully nonlinear strategy for controlling run-andtumble motion depending on the noisy sensing of a ligand gradient. The derived optimal strategy comprises optimal filtering dynamics to estimate the run direction from the noisy sensory input and control function to regulate the motor output. Furthermore, we show that this optimal strategy can be naturally associated with a standard biochemical model and experimental data of the chemotaxis of Escherichia coli. Our results demonstrate that our theoretical framework can be used as a basis for analyzing the efficiency and optimality of run-and-tumble chemotaxis.
\end{abstract}

DOI: 10.1103/PhysRevResearch.4.013120

\section{INTRODUCTION}

A wide variety of organisms, from animals to single cells, are capable of searching for odor sources. These abilities are essential for finding food, suitable environments, and mates, and the search strategies of organisms are subject to selection pressures and are expected to be optimized evolutionarily. Thus, efficient or optimal search strategies have been explored for various living organisms [1-3]. One of the most intensively investigated strategies is the run-and-tumble motion in the chemotaxis of Escherichia coli, whose biochemical signaling pathways have also been elucidated [4].

E. coli can climb up a spatial ligand gradient by sensing temporal changes in the ligand concentration and accordingly regulating the motor (Fig. 1(a)). The motor switches between run and tumble states, resulting in repeated ballistic swimming (run) interrupted by random changes in direction (tumble). By inhibiting tumbles when sensing an increase in ligand concentration and vice versa, E. coli can selectively enhance positive displacement along the gradient's increasing direction. Such a sensory-motor cycle has been theoretically

\footnotetext{
*Also at Institution of Industrial Science, The University of Tokyo, 4-6-1, Komaba, Meguro-ku, Tokyo 153-8505, Japan; and at Universal Biology Institute, The University of Tokyo, 7-3-1, Hongo, Bunkyo-ku 113-8654, Japan; tetsuya@mail.crmind.net

Published by the American Physical Society under the terms of the Creative Commons Attribution 4.0 International license. Further distribution of this work must maintain attribution to the author(s) and the published article's title, journal citation, and DOI.
}

modeled in E. coli chemotaxis [5-9] based on biochemical models of the intracellular signaling pathway [10-16].

To discuss how well E. coli is structured to perform chemotaxis, it is important to understand its sensory-motor cycle in terms of optimality. While biochemical models describe how the actual biochemical pathway behaves, optimality models aim to characterize the best possible chemotactic performance under physical constraints and the strategy that achieves the performance. By comparing the optimality models with the $E$. coli chemotactic responses measured experimentally $[13,17-$ 21], one can understand how close to the physical limit a cell can perform chemotaxis and whether its biochemical signaling pathway is organized in a reasonable way to achieve efficient chemotaxis. Previous attempts using optimality models can be classified into two directions. One uses optimal control models by focusing on the optimal strategy to modulate the tumble rate based on sensing signals. This approach formulates the tumble rate as a functional of sensing signals and considers the optimization of functional with respect to chemotactic performance, such as the gradient climbing speed [22-25]. The other uses optimal filtering models by focusing on the optimality to sensory noise. Because sensory noise cannot be ignored at the cellular level, the biochemical signaling pathway of $E$. coli is expected to possess noiseimmune properties. By deriving an optimal filtering strategy that extracts the ligand concentration or its changes from the noisy signal, we can discuss how well the $E$. coli biochemical signaling pathway is designed to decode information [26-30].

Despite the progress in optimality models, there is still no theory that properly deals with the "sensory-motor cycle," "nonlinear response," and "sensory noise" at the same time 
(a)

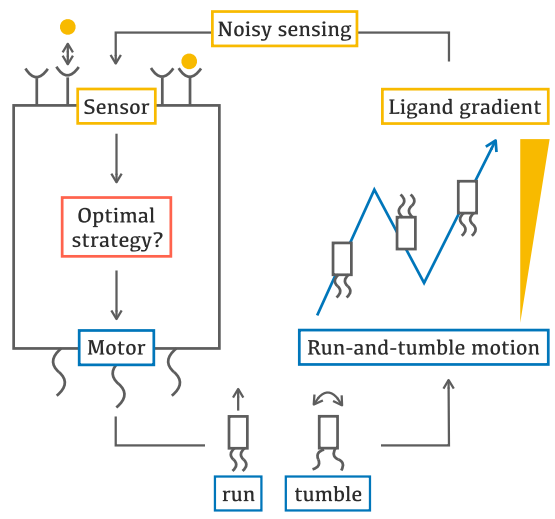

(b)

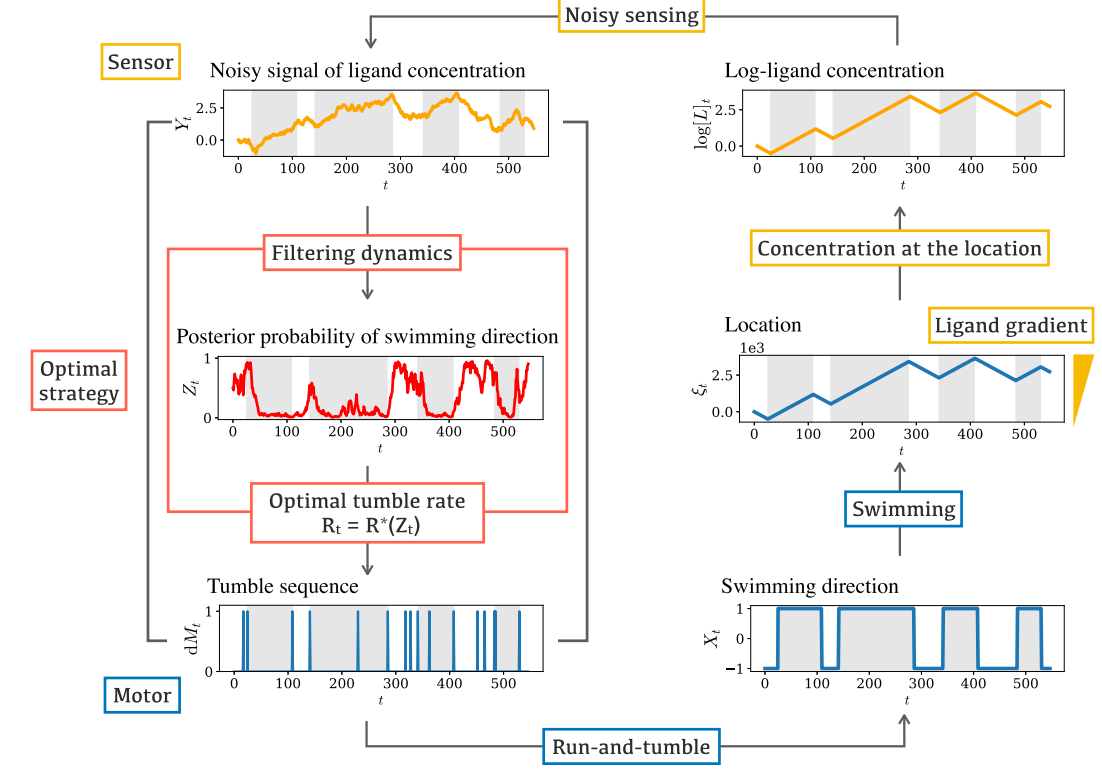

FIG. 1. (a) Schematic diagram of the sensory-motor cycle in E. coli chemotaxis. (b) Sample paths of the variables involved in the cycle driven by the optimal strategy. Each panel shows the tumble event sequence $M_{t}$, swimming direction $X_{t}$, location $\xi_{t}$, ligand concentration on a $\operatorname{logarithmic}$ scale $\log [L]_{t}$, sensing signal $Y_{t}$, and the posterior probability of the swimming direction $Z_{t}$. The gray regions represent the time intervals during which the swimming direction is up the gradient, that is, $X_{t}=+1$. These trajectories were simulated using the dimensionless parameter values $\tilde{\beta}=10^{0.6}, \tilde{R}^{0}=10^{0.4}$, and $\tilde{\lambda}=10^{0.5}$. In the context of $E$. coli chemotaxis, these dimensionless parameter values can correspond to the following biologically realistic dimensional parameter values: $v=20 \mu \mathrm{m} \mathrm{s}^{-1}, c=10^{-3} \mu \mathrm{m}^{-1}, \sigma=8.7 \times 10^{-3} \mathrm{~s}^{-1}$, $\gamma=9.2 \times 10^{-3} \mathrm{~s}^{-1}, \beta=1.8 \times 10^{-3} \mu \mathrm{m}^{-1}$, and $R^{0}=2.3 \times 10^{-2} \mathrm{~s}^{-1}$.

from the perspective of optimality. The optimality of the entire sensory-motor cycle is crucial because the sensing strategy shapes the information available for motor control and the motor control strategy affects what is sensed through motion modulation. However, previous optimal filtering models [26-30] have neglected the influence of motor control on sensing signals. In addition to motor control, previous optimality models have failed to include the nonlinear responses observed (for example, in the biochemical pathway of $E$. coli chemotaxis [16,21]). They considered only linear responses and derived the optimal strategy in that class by assuming a weak ligand gradient [22-25]. Moreover, sensory noise has been neglected in the derivation of those optimal control models. Several theoretical studies have addressed the optimality of the sensory-motor cycle in the presence of nonlinearity and/or noise [7,31-40]. However, it is generally difficult to analytically investigate the effects of noise and nonlinearity; therefore, such effects are often treated only by simulations. A few of these studies analytically treat the impact of noise and nonlinearity on the optimality; however, they do so at the parameter level by assuming fixed structures of strategies. To determine whether the actual biochemical pathway of $E$. coli is structurally optimized, it is important to include a sufficiently large class of strategies as candidates for an optimal strategy.

In this article, we propose an optimality model that considers the "sensory-motor cycle," "nonlinear response," and "sensory noise" integratively based on the framework of a partially observable optimal control [41]. We derive the optimal strategy for tumble rate regulation and show that the strategy is implemented as a combination of two separate components: An optimal filtering dynamics that yields an estimate of the swimming direction and an optimal control function that converts the estimate to the tumble rate. We show that the obtained optimal strategy can be related to both the biochemical model as well as the experimental data of the E. coli signaling pathway. Finally, we discuss possible extensions of the optimality model.

\section{MODELING AND FORMULATION}

To consider a minimal setting, we focus on a control strategy to regulate the run-and-tumble motion on a onedimensional axis along a spatial ligand gradient (Fig. 1(b)). We model a tumble as an instantaneous event that occurs according to the Poisson process with rate $R_{t} \geqslant 0$ and denote by $M_{t} \in \mathbb{N}$ the total number of tumble events up to time $t$. We do not explicitly include the dispersal of swimming direction during the run phase and assume that $E$. coli changes its direction only at tumble events (see Supplemental Material, SM [42] for the case in which the direction can change independently of controlled tumble events, which includes Refs. [43-48]). We denote by $X_{t} \in\{+1,-1\}$ the swimming direction and assume that $X_{t}$ flips with probability $1 / 2$ every time a tumble occurs (see SM for a more general case where the directional change occurs with other probabilities [42]). With these assumptions, we can express the time evolution of the probability of $X_{t}$ under a given tumble rate $R_{t}$ using a continuous-time Markov chain:

$$
\frac{d p_{t}}{d t}=\frac{1}{2} R_{t}\left(\begin{array}{cc}
-1 & 1 \\
1 & -1
\end{array}\right) \boldsymbol{p}_{t},
$$


where $\boldsymbol{p}_{t}:=\left(\mathbb{P}\left(X_{t}=+1\right), \mathbb{P}\left(X_{t}=-1\right)\right)^{\mathbb{T}}$, and the initial condition is set to $\mathbb{P}\left(X_{t}=-1\right)=\pi \in[0,1] . \xi_{t} \in \mathbb{R}$ denotes the position of $E$. coli, and $E$. coli is assumed to move at a constant speed $v$ in the current direction, $d \xi_{t} / d t=v X_{t}$. Note that if the tumble rate $R_{t}$ is constant, there is no net displacement on average because the transition matrix of the direction is symmetric.

Under this setting, we consider the optimal strategy for regulating the tumble rate $R_{t}$ to climb a spatial ligand gradient depending on the noisy sensing of the environment. We introduce sensory noise by defining a sensing signal $Y_{t}$ and specify the available information for the regulation of $R_{t}$ as follows:

$$
\begin{gathered}
R_{t}=\mathcal{R}_{t}^{\mathrm{c}}\left[Y_{0: t}\right], \\
Y_{t}:=h\left(\xi_{t}\right)+\sqrt{\sigma} W_{t},
\end{gathered}
$$

where $Y_{0: t}:=\left\{Y_{t^{\prime}} \mid t^{\prime} \in[0, t]\right\} . \mathcal{R}_{t}^{\mathrm{c}}$ is a control functional representing the strategy of tumble regulation, which can explicitly depend on time. $h$ is a function that represents the ligand-dependent part of the signal, which will be specified later. $W_{t}$ is a standard Wiener process that models the sensory noise, and $\sigma>0$ represents the noise intensity. Note that we do not restrict the candidates of strategies to those realized by a linear response. Of all possible control functionals, we aim to find the optimal control functional $\mathcal{R}_{t}^{*}$, satisfying the following maximization problem:

$$
\mathcal{R}_{0: \infty}^{*}:=\underset{\mathcal{R}_{0: \infty}^{\mathrm{c}}}{\operatorname{argmax}} \mathcal{J}\left(\pi, \mathcal{R}_{0: \infty}^{\mathrm{c}}\right),
$$

where $\mathcal{J}$ is a utility functional defined as follows:

$$
\mathcal{J}\left(\pi, \mathcal{R}_{0: \infty}^{\mathrm{c}}\right):=\mathbb{E}^{\mathrm{c}}\left[\int_{0}^{\infty} e^{-\gamma t} v X_{t} d t\right]-\frac{1}{\beta} \mathcal{C}\left[\mathcal{R}_{0: \infty}^{\mathrm{c}}\right] .
$$

The first term is the temporal integration of velocity $v X_{t}$ and measures the extent to which it climbs a spatial ligand gradient. In the second term, we introduce a control cost $\mathcal{C}$ to obtain a bounded control functional and consider the possible physical cost of tumble regulation. Therefore, the optimization in Eq. (4) represents the problem of how far up the gradient an organism can climb while keeping the cost low. $\mathbb{E}^{\mathrm{c}}$ indicates that the expectation is taken under the controlled process generated by Eqs. (1)-(3) with the initial condition $\mathbb{P}\left(X_{0}=-1\right)=\pi .1 / \beta>0$ and $\gamma>0$ represent the weight of the control cost and the temporal discounting rate, respectively (see SM for another interpretation of $1 / \beta$ [42]). By introducing a temporal discounting factor, the optimization takes greater account of the impact of control in the near future than that in the distant future, and we can interpret the reciprocal of discounting rate as the effective time interval over which the optimization considers (see SM for the interpretation of temporal discounting based on random time interval [42]). Biologically, it is reasonable to assume that organisms are optimized to take greater account of near-future impacts because the impact of current control should decrease over time owing to rotational diffusion or changes in the environment. We consider that the temporal discounting rate is set according to the time scale of these factors. Temporal discounting also has a technical advantage, making it easier to investigate optimal control both analytically and numerically [49].
Defining the control cost is nontrivial, especially for biological systems. While quadratic cost functions are conventionally used in control theory, a quadratic form of $\mathcal{C}\left[\mathcal{R}_{0: t}^{\mathrm{c}}\right]$ may not admit a natural interpretation. In addition, $\mathcal{C}\left[\mathcal{R}_{0: t}^{\mathrm{c}}\right]$ should be designed appropriately to guarantee that the derived optimal control functional does not produce biologically irrelevant values such as $R_{t}<0$ or $R_{t}=0$. To deal with this difficulty, we introduce a control cost using the framework of Kullback-Leibler (KL) control. The KL control framework assumes an uncontrolled process and defines the control cost by the discrepancy between the controlled and uncontrolled processes in terms of the $\mathrm{KL}$ divergence, $\mathcal{D}_{\mathrm{KL}}\left[\mathbb{P}^{\mathrm{c}} \| \mathbb{P}^{0}\right]$, where $\mathbb{P}^{\mathrm{c}}$ and $\mathbb{P}^{0}$ denote the path probabilities of the controlled and uncontrolled processes of tumbling causally conditioned on the sensing signals, respectively. We model an uncontrolled tumbling process using a reference constant-rate process, that is, $R_{t}^{0}=R^{0}>0$. Because the constant rate Poisson process is the simplest and least predictable, it can work as a representative of an uncontrolled situation. With a temporal discounting factor, we derive the control cost functional under our setting (see SM [42] for derivation and the relation to the information flow discussed in Ref. [25]):

$$
\mathcal{C}\left[\mathcal{R}_{0: \infty}^{\mathrm{c}}\right]:=\mathbb{E}^{\mathrm{c}}\left[\int_{0}^{\infty} e^{-\gamma t}\left\{\log \frac{R_{t}}{R^{0}} d M_{t}-\left(R_{t}-R^{0}\right) d t\right\}\right] .
$$

We can derive the optimal control functional $\mathcal{R}_{0: \infty}^{*}$ in Eq. (4) based on the framework of the partially observable control [41] (see SM for derivation [42]). Even though we assume that the tumble rate $R_{t}$ at each time $t$ can depend on the entire history of the sensing signal, $Y_{0: t}$, we can prove that the optimal control under a certain condition is achieved only by using the posterior probability of the direction $Z_{t}:=$ $\mathbb{P}\left(X_{t}=-1 \mid Y_{0: t}\right)$, which summarizes the information contained in $Y_{0: t}$. In particular, if the function $h$ in the sensing signal [Eq. (3)] is affine, that is, $h\left(\xi_{t}\right)=\lambda \xi_{t}+$ const., where $\lambda$ is a constant, the optimal control functional is expressed by the following optimal control function $R^{*}$, which takes $Z_{t}$ as its argument:

$$
\mathcal{R}_{t}^{*}\left[Y_{0: t}\right]=R^{*}\left(Z_{t}\right):=R^{0} \exp \left\{-\tilde{\beta}\left(Z_{t}-\frac{1}{2}\right) \frac{d \tilde{V}}{d Z}\left(Z_{t}\right)\right\} .
$$

Here, $\tilde{V}$ is a scaled value function on $[0,1]$ that satisfies $\tilde{V}(\pi)=\max _{\mathcal{R}_{0: \infty}^{\mathrm{c}}} \tilde{\mathcal{J}}\left(\pi, \mathcal{R}_{0: \infty}^{\mathrm{c}}\right)$, where $\tilde{\mathcal{J}}:=(\gamma / v) \mathcal{J}$ is a scaled utility functional. $\tilde{V}$ is obtained by solving the following second-order differential equation, known as the Hamilton-Jacobi-Bellman (HJB) equation, in the optimal control theory:

$$
\begin{aligned}
\tilde{V}= & \frac{\tilde{R}^{0}}{\tilde{\beta}}\left[\exp \left\{-\tilde{\beta}\left(Z-\frac{1}{2}\right) \frac{d \tilde{V}}{d Z}\right\}-1\right] \\
& +\{\tilde{\lambda} Z(1-Z)\}^{2} \frac{d^{2} \tilde{V}}{d Z^{2}}-(2 Z-1),
\end{aligned}
$$

where $\tilde{R}^{0}:=R^{0} / \gamma, \tilde{\lambda}:=\sqrt{2} \lambda v / \sqrt{\sigma \gamma}$, and $\tilde{\beta}:=\beta v / \gamma$ are dimensionless parameters that describe the ratio between the temporal discounting rate and the reference tumble rate, the signal-to-noise ratio of the sensory signal $Y_{t}$, and the relative weight of the net displacement with respect to the control cost, respectively. 
We can show that the information needed for optimal control $Z_{t}$ can be calculated sequentially and separately before feeding it into the optimal control function [Eq. (6)]. Based on the theory of nonlinear filtering, we derived the time evolution of $Z_{t}$ as the following stochastic differential equation:

$$
\frac{d Z_{t}}{d t}=-R^{*}\left(Z_{t}\right)\left(Z_{t}-\frac{1}{2}\right)-K Z_{t}\left(1-Z_{t}\right) \circ \frac{d Y_{t}}{d t} .
$$

Here, o represents the Stratonovich integral. The first and the second terms represent the prediction based on prior knowledge of the directional change represented by Eq. (1) and the update of the posterior based on the sensing signal [Eq. (3)], respectively, [30]. $K$ and the initial state of $Z_{t}$ should be adjusted to $K=2 \lambda v / \sigma$ and $Z_{0}=\pi$ such that the dynamics of $Z_{t}$ are compatible with the optimal control function.

From the above discussion, we obtain the optimal strategy for tumble regulation as a combination of the filtering dynamics [Eq. (9)] of the sensing signal and the optimal control function [Eq. (6)] driven by the filtered signal $Z_{t}$ (Fig. 1(b)).

\section{COMPARISON AND CORRESPONDENCE WITH THE BIOCHEMICAL MODEL}

To relate the optimal but abstract strategy represented by Eqs. (9) and (6) to the actual biochemical pathway of E. coli, we introduce two additional assumptions about the sensing process. First, we assume that the ligand concentration $[L]_{t}$ sensed by $E$. coli is exponentially distributed along the $\xi$ axis, $[L]_{t} \propto \exp \left(c \xi_{t}\right)$, where $c$ represents the steepness of the gradient. Second, we assume that the sensory noise is Gaussian in $\log$ concentration $Y_{t}=\log [L]_{t}+\sqrt{\sigma} W_{t}$. The setting specified by these two assumptions satisfies the condition that $h$ in the sensing signal [Eq. (3)] is affine with the parameter set to $\lambda=c$.

Under this setting, we transform Eq. (9) into a form that clarifies its relation with a biochemical model of the $E$. coli signaling pathway [16]. Following a previous paper [30], we define the $\log$-likelihood ratio as $\theta_{t}:=\log \left\{\left(1-Z_{t}\right) / Z_{t}\right\}$ and introduce an additional variable $\mu_{t}$, called a prediction term. Then, we can derive the following equivalent expression of the filtering dynamics Eq. (9):

$$
\begin{gathered}
Z_{t}=\frac{1}{1+\exp \left(\theta_{t}\right)}, \\
\theta_{t}=-\kappa \mu_{t}+K\left[\log [L]_{t}+\sqrt{\sigma} W_{t}\right]+\phi, \\
\frac{d \mu_{t}}{d t}=-\frac{R^{*}\left(Z_{t}\right)}{\kappa} \frac{Z_{t}-1 / 2}{Z_{t}\left(1-Z_{t}\right)}=: F_{\mathrm{OPT}}\left(Z_{t}\right),
\end{gathered}
$$

where $\kappa>0$ is an arbitrary constant and $\phi:=\log \{(1-$ $\pi) / \pi\}-K \log [L]_{0}+\kappa \mu_{0}$ is a constant of the integral (see SM for derivation [42]).

Next, we briefly review the standard biochemical model of the E. coli signaling pathway represented by Eqs. (13)-(15), which are subsequently compared with Eqs. (10)-(12). In an $E$. coli cell, the ligand is sensed by an array of receptors, each of which takes either an active or inactive state, and the active receptors increase the rate of the tumbles by mediating proteins. The biochemical model defines and quantifies the receptor activity $a_{t}$ by the ratio of active receptors. The receptors are also influenced by their methylation, which is quantified in the model by the average methylation level $m_{t}$. The dependence of $a_{t}$ on $m_{t}$ and on the ligand concentration $[L]_{t}$ is described by the Monod-Wyman-Changeux (MWC) allosteric model:

$$
\begin{gathered}
a_{t}=\frac{1}{1+\exp \left(f_{t}\right)}, \\
f_{t}=N\left(-\alpha m_{t}+\log [L]_{t}+I\right),
\end{gathered}
$$

where $f_{t}$ is the free energy difference between the active and inactive states. $N, \alpha$, and $I$ are biochemical parameters. The receptor activity $a_{t}$ affects the methylation and demethylation enzymes CheR and CheB, and the resultant kinetics of methylation is modeled as

$$
\frac{d m_{t}}{d t}=F\left(a_{t}\right)
$$

where $F$ is a decreasing function of $[0,1]$ with a single zero point $\bar{a}$. We can see that the kinetics represented by $F$ works as a negative feedback on $a_{t}$ by noting that $\partial a_{t} / \partial m_{t}>0$ and $F^{\prime}\left(a_{t}\right)<0$ hold. The dependence of the tumble rate on the state of the receptors can be modeled by setting the tumble rate as an increasing function of $a_{t}[5,6,8,9,50]$. The biochemical model composed of Eqs. (13)-(15) can describe the experimental responses of $E$. coli to various ligand profiles [16,51].

We can now see that the biochemical quantities $\left(a_{t}, f_{t}, m_{t}\right)$ in Eqs. (13)-(15) have the same kinetic structure as the filtering quantities $\left(Z_{t}, \theta_{t}, \mu_{t}\right)$ in Eqs. (10)-(12). The posterior probability $Z_{t}$ and the receptor activity $a_{t}$ are described by the sigmoidal transformation of $\theta_{t}$ and $f_{t}$, respectively. The $\log$-likelihood ratio $\theta_{t}$ and the free energy difference $f_{t}$ are expressed by the sum of $\log [L]_{t}, \mu_{t}$, and $m_{t}$. The dynamics of the prediction term $\mu_{t}$ and the methylation level $m_{t}$ are described by functions of $Z_{t}$ and $a_{t}$, respectively. The tumble rate is modeled by the functions $Z_{t}$ and $a_{t}$ in the optimal and biochemical models, respectively. This correspondence between the optimal and biochemical models suggests that the E. coli biochemical signaling pathway possesses a desirable structure for the optimal control of tumble regulation under sensory noise. It should be noted that even though we do not include other stochasticities in the biochemical reactions than the receptors in our optimality modeling, from the correspondence of Eqs. (11) and (14), the optimality model can effectively accommodate the noise of methylation reaction by adding that effect to $\sqrt{\sigma} W_{t}$ because $m_{t}$ and $\sqrt{\sigma} W_{t}$ appear additively in Eq. (14).

To see whether the dependence of the tumble rate on $Z_{t}$ and $a_{t}$ is consistent between the optimal and biochemical models, we investigated the functional form of the optimal control function $R^{*}\left(Z_{t}\right)$. We calculate $R^{*}$ by substituting into Eq. (6) the value function, $\tilde{V}$, obtained by numerically solving the HJB equation [Eq. (8)]. In Fig. 2(a), we show the optimal control function for a representative set of parameter values. It can be seen that $R^{*}(Z)$ monotonically increases with $Z$ and is greater than $R^{0}$ for $Z>1 / 2$ and smaller than $R^{0}$ for $Z<1 / 2$. As shown in Fig. 4, this property of $R^{*}$ is robust to changes in parameter values (see also SM for the detail of the functional form of $R^{*}$ ). The result indicates that the tumble rate should be high when the direction is likely to be down the gradient and vice versa to perform chemotaxis optimally under noise. This property of the optimal control function is consistent with the 

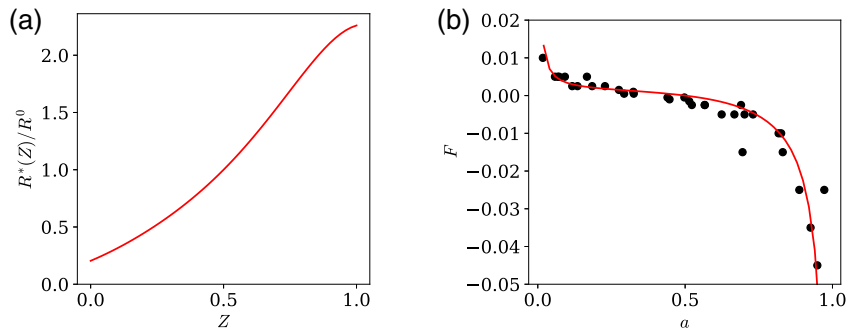

FIG. 2. (a) Numerical solution of the optimal control function $R^{*}$. (b) A comparison between the feedback function of the optimality model, $F_{\text {OPT }}$ (red curve) and that of the experimental data, $F_{\text {exp }}$, (black points) [21]. The parameter values in $R^{*}$ and $F_{\text {OPT }}$ are set to $R^{0}=2.3 \times 10^{-2}, \tilde{\beta}=10^{0.6}, \tilde{R}^{0}=10^{0.4}$, and $\tilde{\lambda}=10^{0.5}$ (see SM for the fitting procedure [42]).

regulation of the tumble rate in cells of $E$. coli, which can be modeled by an increasing function of $a[5,6,8,9]$.

Next, we clarify whether the optimal feedback function, $F_{\mathrm{OPT}}(Z)=-\left\{R^{*}(Z) / \kappa\right\}(Z-1 / 2) /\{Z(1-Z)\}$, in Eq. (12) explains the corresponding feedback function $F$ in Eq. (15), which describes the methylation kinetics. Although the biochemical mechanism governing the kinetics is not fully understood, the functional form of $F$ is experimentally estimated using exponential ramp-response measurements [21]. We compare $F_{\text {OPT }}$ with the experimentally measured feedback function $F_{\text {EXP. We obtain }} F_{\text {OPT }}$ by substituting a numerical solution of $R^{*}$ into the equation of $F_{\mathrm{OPT}}$ and fit $F_{\mathrm{OPT}}$ to $F_{\mathrm{EXP}}$ by adjusting $\tilde{\beta}, \tilde{R}^{0}$, and $R^{0}$ (see SM for details of the fitting procedure [42]). Figure 2(b) shows the functional form of $F_{\mathrm{OPT}}$ and the experimental data, $F_{\mathrm{EXP}}[21] . F_{\mathrm{OPT}}$ explains $F_{\text {EXP }}$ well, particularly its characteristic nonlinearity, the gentle slope around $a=1 / 2$, and the steep slope near $a=1$. The steep slope near $a=1$ is conjectured to be generated by CheB phosphorylation, and its functional role has not been completely clarified $[21,31,52]$. The agreement between $F_{\text {OPT }}$ and $F_{\mathrm{EXP}}$ indicates that the methylation kinetics represented by $F_{\text {EXP }}$ can be understood as a property of optimal filtering incorporating the prediction of motor regulation.

We should note that, in our previous paper, we related $F_{\text {EXP }}$ to the feedback function obtained without considering motor control [30]. The feedback function there was $F_{\mathrm{OPT}}(z)=$ $-(R / \kappa)(z-1 / 2) /\{z(1-z)\}$, where $R^{*}(z)$ in Eq. (12) is replaced with a constant $R$. Because this function is symmetric with respect to $z=1 / 2$, whereas $F_{\mathrm{EXP}}$ is asymmetric, a change of the variable for $Z$ had to be introduced to fit $F_{\mathrm{OPT}}$ to $F_{\mathrm{EXP}}$ in the previous study. In this study, the effect of the control $R^{*}(Z)$ accounts for the asymmetry of the function without such a change of variable. Thus, we have obtained a more natural explanation of $F_{\text {EXP }}$ by considering optimal motor control.

Even after considering motor control, there is still a discrepancy between the zero-point of $F_{\mathrm{OPT}}(z=1 / 2)$ and the previously estimated zero point of $F_{\mathrm{EXP}}(a \approx 0.3)$. We think that there are at least three possible causes of the discrepancy. The first is that $a_{t}$ and $Z_{t}$ may not directly correspond. The optimal control model can be transformed by adding a constant to $\theta_{t}$ as done in [30], with which the zero point of $F_{\mathrm{OPT}}$ shifts without breaking the correspondence between the biochemical model and the optimal control model. The
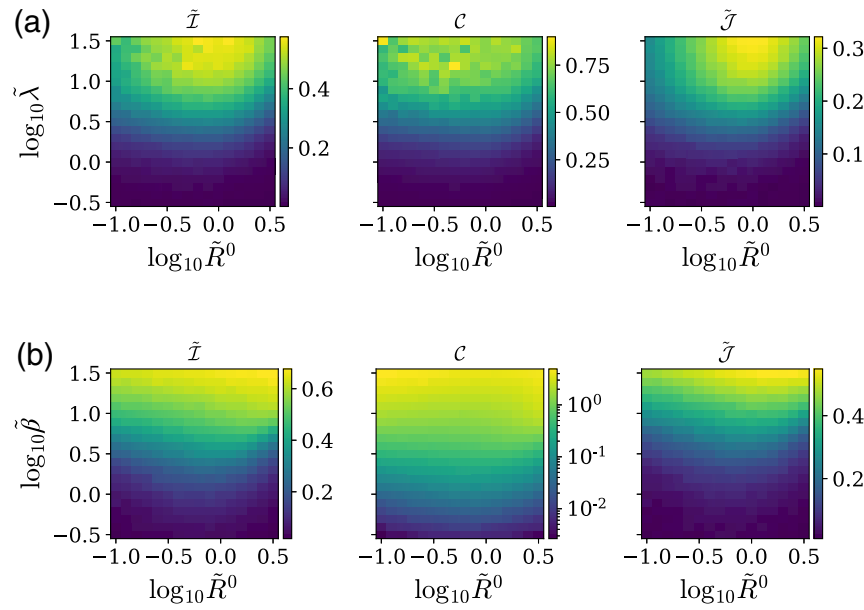

FIG. 3. Dependence of the net displacement, $\tilde{\mathcal{I}}=\int_{0}^{\infty} e^{-\tau} X_{\tau} d \tau$, the control cost $\mathcal{C}$, and the utility $\tilde{\mathcal{J}}=\tilde{\mathcal{I}}-\mathcal{C} / \tilde{\beta}$ on $\tilde{\lambda}$ and $\tilde{R}^{0}$ (a) and on $\tilde{\beta}$ and $\tilde{R}^{0}$ (b). We used the parameter values, $\tilde{\beta}=10^{0.5}, \tilde{R}^{0}=$ $1, \tilde{\lambda}=10^{0.5}$, except for those designated in each panel.

second possibility is that the zero-point estimated from the data has a statistical variation. Estimating the zero point can be a difficult problem due to the gentle slope of $F$ around the zero point. The last is that the experimental estimation of $F$ may be biased because the sensory noise was ignored in the previous research [21] (see SM for detail [42]). Further analysis of the experimental data is needed to clarify the cause of the discrepancy.

\section{PARAMETER DEPENDENCY OF CONTROL FUNCTION AND PERFORMANCE}

The results described above indicate that the biochemical pathway of E. coli can be understood as a physical implementation of the optimal strategy under sensory noise.

We then explore how the optimal control strategy and its chemotactic performance depend on conditions such as the (a)

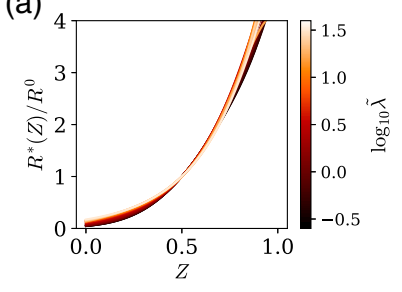

(b)

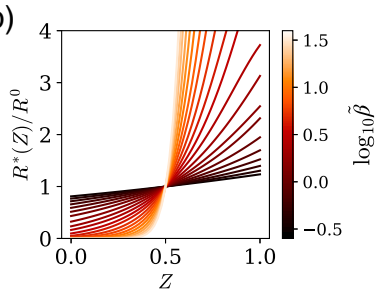

(c)

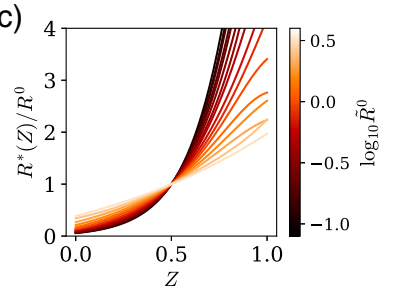

FIG. 4. Dependence of normalized optimal control function $R^{*}(Z) / R^{0}$ on parameters $\tilde{\lambda}$ (a), $\tilde{\beta}(\mathrm{b})$, and $\tilde{R}^{0}(\mathrm{c})$. We used the parameter values $\tilde{\beta}=10^{0.5}, \tilde{R}^{0}=1, \tilde{\lambda}=10^{0.5}$, except for those designated in each panel. 
signal-to-noise ratio using numerical simulations under various parameters. Of the control function [Eq. (6)] and the filtering dynamics [Eq. (9)] that comprise the optimal strategy, we focus only on the parameter dependence of the normalized optimal control function, $R^{*}(Z) / R^{0}=\exp \{-\tilde{\beta}(Z-$ $1 / 2) \partial \tilde{V} / \partial Z)\}$ [Eq. (6)] (see Ref. [30] and SM for the parameter dependence of the filtering dynamics, Eq. (9)). To quantify the chemotactic performance, we introduce three dimensionless performance indices: the scaled net displacement along the ligand gradient $\tilde{\mathcal{I}}:=\mathbb{E}\left[\int_{0}^{\infty} e^{-\tau} X_{\tau} \mathrm{d} \tau\right]$, the control $\operatorname{cost} \mathcal{C}$, and the scaled utility $\tilde{\mathcal{J}}:=(\gamma / v) \mathcal{J}=\tilde{\mathcal{I}}-\mathcal{C} / \tilde{\beta}$ where we define $\tau:=\gamma t$. We note that the function $R^{*}(Z) / R^{0}$ and the performance indices $\tilde{\mathcal{I}}, \mathcal{C}$, and $\tilde{\mathcal{J}}$ are determined only by the dimensionless parameters $\tilde{\beta}, \tilde{R}^{0}$, and $\tilde{\lambda}$, which appear in the HJB equation [Eq. (8)]. We plot the parameter dependence of the performance indices and normalized optimal control function as shown in Figs. 3 and 4, respectively (see also SM for the plot of maximum net displacement $\tilde{\mathcal{I}}$ as a function of control cost $\mathcal{C}$ ).

We first investigate whether a high signal-to-noise ratio improves the net displacement and how the control strategy changes depending on the dimensionless signal-to-noise ratio, $\tilde{\lambda}$. From Fig. 3(a), we can see that in a high signal-to-noise ratio situation, a large net displacement can be obtained at the expense of a high control cost, as both the net displacement $\tilde{\mathcal{I}}$ and the control cost $\mathcal{C}$ become large when $\tilde{\lambda}$ is high. On the other hand, Fig. 4(a) shows that the optimal control function is almost unchanged even though the value of $\tilde{\lambda}$ changes. This phenomenon can be understood by considering that the signalto-noise ratio is reflected in tumble regulation only through the uncertainty of the estimator. If the signal-to-noise ratio is high, the swimming direction can be estimated with high precision to be either up $Z_{t} \approx 0$ or down $Z_{t} \approx 1$ the gradient. In contrast, if the signal-to-noise ratio is low, it is difficult to accurately estimate whether the swimming direction is up or down; thus, the estimator tends to take an intermediate value around $Z_{t} \approx 0.5$ (See also the SM for the behavior of $Z_{t}$ under different values of $\tilde{\lambda}$ ). Therefore, even though the functional form of $R^{*} / R^{0}$ is almost invariant to $\tilde{\lambda}$, the actual tumble rate would be modulated drastically under high $\tilde{\lambda}$ and gently under low $\tilde{\lambda}$.

Next, we investigate whether and how the net displacement can be increased by varying the relative weight of the control cost to the net displacement in the utility functional, that is, $1 / \tilde{\beta}$. Figure 3 (b) shows that both the net displacement $\tilde{\mathcal{I}}$ and the control cost $\mathcal{C}$ are increasing functions of $\tilde{\beta}$ and Fig. 4(b) shows that the slope of $R^{*} / R^{0}$ increases with the increase of $\tilde{\beta}$. This result indicates that as the weight of the control cost decreases, the optimal control strategy tends to use abrupt switching of motors to increase the net displacement. If the weight of the control cost is high, the optimal strategy employs moderate switching of the motor. Thus, the steepness of the control function may be used to estimate the potential control costs of the actual biological systems.

Finally, by varying $\tilde{R}^{0}=R^{0} / \gamma$, we investigate how the chemotactic performance and optimal control function depend on the ratio of the time scale of the reference tumbling rate $\left(R^{0}\right)^{-1}$ and the time scale of the utility discounting $\gamma^{-1}$. The timescale of utility discounting indicates how far into the future the optimization will take into account. From Fig. 4(c), we can see that the fold change in the optimal control function decreases with an increase in $\tilde{R}^{0}$, indicating that the tumbling rate needs to be controlled gently to maximize the long-term utility. Figure 3 shows that there is an optimal value of $\tilde{R}^{0}$ that maximizes the net displacement. The existence of the optimum may be understood as a compromise between the speed and accuracy of decision-making under sensory noise. If $\tilde{R}^{0}$ is small, the estimation of the swimming direction can be accurate because the noisy signal can be integrated for a long time before the next tumbling occurs. However, if $\tilde{R}^{0}$ is too small, the initiation of the tumble may be deferred even when the swimming direction is not appropriate. The optimal $\tilde{R}^{0}$ is approximately $\tilde{R}^{0} \approx 1$, irrespective of the other parameters, suggesting that a finite optimal basal tumble rate emerges from the finite temporal discounting rate of the utility. This result is consistent with a previous paper [25] that reported that an optimal basal tumble rate emerges from the rotational diffusion because the temporal discounting rate can be interpreted as the rotational diffusion rate.

In summary, chemotactic performance in terms of the net displacement along the ligand gradient increases at the expense of a large control cost when the $\mathrm{SN}$ ratio is high and the optimal control function has a steep slope. The net displacement also depends on the balance between the time scale for tumbling and that for performance evaluation. The optimal control function is an increasing function of $Z$ in a wide range of parameters, and the steepness of its slope is modulated depending on the parameters $\tilde{\beta}$ and $\tilde{R}^{0}$; however, it is almost independent of $\tilde{\lambda}$.

\section{SUMMARY AND DISCUSSION}

In this work, we derived an optimal sensing and motorcontrol strategy under sensory noise, which characterizes the performance limit of general run-and-tumble chemotaxis. We also investigated the connection between the derived optimal strategy and a well-characterized biochemical model of the signaling pathway of $E$. coli. By explicitly considering motor control and its optimality, we verified that $E$. coli model and data can be related to the structure of the optimal strategy more naturally and reasonably. This result reinforces the idea that $E$. coli exploits the structure of the optimal strategy that is necessary for attaining the performance limit [30].

To verify the connection more quantitatively with other experimental data, we need to study the motor control in a more detailed manner because the proposed optimality model cannot be compared directly with the currently available experimental data of $E$. coli motor control. The motor state of E. coli is experimentally measured by a clockwise (CW) bias [50]. To define the $\mathrm{CW}$ bias, the duration of tumble should be finite; however, we derived the optimality model under the approximation that the duration of tumble is infinitely small. One way to alleviate this problem is to experimentally measure the frequency of tumble as a function of receptor activity, which can be compared directly with our results. The other way is to model the motor control as a stochastic switching between run and tumble $[5,6,9]$ such that we can compare the model with the $\mathrm{CW}$ bias data. To this end, the proposed model can be extended by considering a Markov chain with four states, $\left(X_{t}, \tilde{M}_{t}\right)$, where $X_{t} \in\{+1,-1\}$ is the swimming 
direction and $\tilde{M}_{t} \in\{$ run, tumble $\}$ is the motor state. Such an extension may enable us to further verify if the optimality model can explain the experimental data of motor control.

With the above extension, we may discuss the optimality of a broader class of exploratory behaviors observed in other organisms [53,54]. For example, Drosophila larvae show head casting behavior in which gradients can be sensed without moving [54]. Such behavior can be treated by adding a head cast to the motor state $\tilde{M}$ and by modeling the velocity and sensing signal appropriately during the head cast. It would be interesting to see how the optimal frequency and duration of the head cast depend on conditions such as the signal-to-noise ratio in the run and head cast states.

Another possible direction is to extend sensory models to multiple ligands. In this study, we assumed that there is a single type of attractant ligand; however, there may be many types of ligands that work as attractants or repellents with different importance [55-57]. We may incorporate the sensing of multiple ligands by modeling the gradient of each ligand and extending the sensing signal $Y_{t}$ to a vector-valued Wiener process. The importance of different ligands may temporally change depending on the internal state of the cell, such as nutritional requirements [58]. It would be interesting to consider not only the regulation of the motor but also the integration of sensory information. By changing the sensory integration based on nutritional requirements, a cell may focus its attention on the gradients of important nutrients. In addition, generally, there is cross talk between receptors. By modeling the sensing signal appropriately, we may discuss the effect of the cross talk on the performance of sensing and motor control.

In the extensions described so far, the estimated state is limited to finite states. However, actual organisms move in a two-dimensional space or in more complex environments and may need to estimate the direction and other environmental factors as continuous quantities. The posterior distribution of even a single continuous quantity cannot be represented by a finite vector in general, and it is difficult to implement its storage and update strictly in living organisms. To effectively deal with such difficulties with limited capacity and memory, organisms may internally represent complex environments by simpler approximate models. To discuss the optimal control based on approximate representations, we may need to consider other formulations, such as the active inference framework, which uses variational approximation of posterior distributions [59]. Also, there may be different ways to incorporate an environmental factor in approximate representation. For example, we explained that the effect of rotational diffusion can be incorporated through the temporal discounting factor, but it may also be incorporated by modifying the model of cell motion. To address the problem of which representation is more advantageous and is actually used by organisms, we need to further develop the formulation of optimization problem and compare it with various experimental results.

The combination of optimal filtering and KL control for partially observed systems can work as a theoretical basis for all these extensions as well as for analyzing the efficiency and optimality of various chemotactic phenomena.

\section{ACKNOWLEDGMENTS}

We would like to thank Keita Kamino for a fruitful discussion. The first author received a JSPS Research Fellowship (Grant No. 20J21362). This research was supported by JSPS KAKENHI (Grant No. 19H05799) and JST CREST (Grant No. JPMJCR2011).
[1] O. Bénichou, C. Loverdo, M. Moreau, and R. Voituriez, Intermittent search strategies, Rev. Mod. Phys. 83, 81 (2011).

[2] A. M. Hein, F. Carrara, D. R. Brumley, R. Stocker, and S. A. Levin, Natural search algorithms as a bridge between organisms, evolution, and ecology, Proc. Natl. Acad. Sci. USA 113, 9413 (2016).

[3] K. L. Baker, M. Dickinson, T. M. Findley, D. H. Gire, M. Louis, M. P. Suver, J. V. Verhagen, K. I. Nagel, and M. C. Smear, Algorithms for olfactory search across species, J. Neurosci. 38, 9383 (2018).

[4] H. C. Berg, E. coli in Motion (Springer, New York, 2004).

[5] N. Vladimirov, L. Løvdok, D. Lebiedz, and V. Sourjik, Dependence of bacterial chemotaxis on gradient shape and adaptation rate, PLoS Comput. Biol. 4, e1000242 (2008).

[6] L. Jiang, Q. Ouyang, and Y. Tu, Quantitative modeling of escherichia coli chemotactic motion in environments varying in space and time, PLoS Comput. Biol. 6 (2010).

[7] A. Celani, T. S. Shimizu, and M. Vergassola, Molecular and functional aspects of bacterial chemotaxis, J. Stat. Phys. 144, 219 (2011).
[8] G. Si, T. Wu, Q. Ouyang, and Y. Tu, Pathway-Based Mean-Field Model for Escherichia coli Chemotaxis, Phys. Rev. Lett. 109, 048101 (2012).

[9] Y. S. Dufour, X. Fu, L. Hernandez-Nunez, and T. Emonet, Limits of feedback control in bacterial chemotaxis, PLoS Comput. Biol. 10, e1003694 (2014).

[10] D. Bray, M. D. Levin, and C. J. Morton-Firth, Receptor clustering as a cellular mechanism to control sensitivity, Nature (London) 393, 85 (1998).

[11] N. Barkai and S. Leibler, Robustness in simple biochemical networks, Nature (London) 387, 913 (1997).

[12] T.-M. Yi, Y. Huang, M. I. Simon, and J. Doyle, Robust perfect adaptation in bacterial chemotaxis through integral feedback control, Proc. Natl. Acad. Sci. USA 97, 4649 (2000).

[13] V. Sourjik and H. C. Berg, Functional interactions between receptors in bacterial chemotaxis, Nature (London) 428, 437 (2004).

[14] J. E. Keymer, R. G. Endres, M. Skoge, Y. Meir, and N. S. Wingreen, Chemosensing in Escherichia coli: Two regimes of two-state receptors, Proc. Natl. Acad. Sci. USA 103, 1786 (2006). 
[15] B. A. Mello and Y. Tu, Effects of adaptation in maintaining high sensitivity over a wide range of backgrounds for Escherichia coli chemotaxis, Biophys. J. 92, 2329 (2007).

[16] Y. Tu, T. S. Shimizu, and H. C. Berg, Modeling the chemotactic response of Escherichia coli to time-varying stimuli, Proc. Natl. Acad. Sci. USA 105, 14855 (2008).

[17] S. M. Block, J. E. Segall, and H. C. Berg, Impulse responses in bacterial chemotaxis, Cell 31, 215 (1982).

[18] S. M. Block, J. E. Segall, and H. C. Berg, Adaptation kinetics in bacterial chemotaxis., J. Bacteriol. 154, 312 (1983).

[19] J. E. Segall, S. M. Block, and H. C. Berg, Temporal comparisons in bacterial chemotaxis, Proc. Natl. Acad. Sci. USA 83, 8987 (1986)

[20] V. Sourjik and H. C. Berg, Receptor sensitivity in bacterial chemotaxis, Proc. Natl. Acad. Sci. USA 99, 123 (2002).

[21] T. S. Shimizu, Y. Tu, and H. C. Berg, A modular gradientsensing network for chemotaxis in Escherichia coli revealed by responses to time-varying stimuli, Mol. Syst. Biol. 6, 382 (2010).

[22] P.-G. De Gennes, Chemotaxis: The role of internal delays, Eur. Biophys. J. 33, 691 (2004).

[23] D. A. Clark and L. C. Grant, The bacterial chemotactic response reflects a compromise between transient and steady-state behavior, Proc. Natl. Acad. Sci. USA 102, 9150 (2005).

[24] A. Celani and M. Vergassola, Bacterial strategies for chemotaxis response, Proc. Natl. Acad. Sci. USA 107, 1391 (2010).

[25] H. Mattingly, K. Kamino, B. Machta, and T. Emonet, Escherichia coli chemotaxis is information limited, Nat. Phys. 17, 1426 (2021).

[26] B. W. Andrews, T.-M. Yi, and P. A. Iglesias, Optimal noise filtering in the chemotactic response of Escherichia coli, PLoS Comput. Biol. 2, e154 (2006).

[27] T. Mora and N. S. Wingreen, Limits of Sensing Temporal Concentration Changes by Single Cells, Phys. Rev. Lett. 104, 248101 (2010).

[28] G. Aquino, L. Tweedy, D. Heinrich, and R. G. Endres, Memory improves precision of cell sensing in fluctuating environments, Sci. Rep. 4, 5688 (2014).

[29] T. Mora and I. Nemenman, Physical Limit to Concentration Sensing in a Changing Environment, Phys. Rev. Lett. 123, 198101 (2019).

[30] K. Nakamura and T. J. Kobayashi, Connection between the Bacterial Chemotactic Network and Optimal Filtering, Phys. Rev. Lett. 126, 128102 (2021).

[31] M. Kollmann, L. Løvdok, K. Bartholomé, J. Timmer, and V. Sourjik, Design principles of a bacterial signalling network, Nature (London) 438, 504 (2005).

[32] Y. Kafri and R. A. da Silveira, Steady-State Chemotaxis in Escherichia coli, Phys. Rev. Lett. 100, 238101 (2008).

[33] M. Flores, T. S. Shimizu, P. R. ten Wolde, and F. Tostevin, Signaling Noise Enhances Chemotactic Drift of E. coli, Phys. Rev. Lett. 109, 148101 (2012).

[34] R. He, R. Zhang, and J. Yuan, Noise-induced increase of sensitivity in bacterial chemotaxis, Biophys. J. 111, 430 (2016).

[35] A. M. Hein, D. R. Brumley, F. Carrara, R. Stocker, and S. A. Levin, Physical limits on bacterial navigation in dynamic environments, J. R. Soc. Interface 13, 20150844 (2016).

[36] J. Long, S. W. Zucker, and T. Emonet, Feedback between motion and sensation provides nonlinear boost in run-and-tumble navigation, PLoS Comput. Biol. 13, e1005429 (2017).

[37] S. Dev and S. Chatterjee, Optimal methylation noise for best chemotactic performance of E. coli, Phys. Rev. E 97, 032420 (2018).

[38] J. B. Kirkegaard and R. E. Goldstein, The role of tumbling frequency and persistence in optimal run-and-tumble chemotaxis, IMA J. Appl. Math. 83, 700 (2018).

[39] A. Gosztolai and M. Barahona, Cellular memory enhances bacterial chemotactic navigation in rugged environments, Commun. Phys. 3, 1 (2020).

[40] S. D. Mandal and S. Chatterjee, Effect of receptor clustering on chemotactic performance of $E$. coli: Sensing versus adaptation, Phys. Rev. E 103, L030401 (2021).

[41] A. Segall, Optimal control of noisy finite-state Markov processes, IEEE Trans. Autom. Control 22, 179 (1977).

[42] See Supplemental Material at http://link.aps.org/supplemental/ 10.1103/PhysRevResearch.4.013120 for derivation of the optimal control and further discussions.

[43] A. H. Jazwinski, Stochastic Processes and Filtering Theory (Courier Corporation, New York, 2007).

[44] H. H. Permuter, Y.-H. Kim, and T. Weissman, Interpretations of directed information in portfolio theory, data compression, and hypothesis testing, IEEE Trans. Inf. Theory 57, 3248 (2011).

[45] G. Kramer, Directed information for channels with feedback, Ph.D. thesis, ETH Zurich,1998.

[46] M. Fujisaki, G. Kallianpur, and H. Kunita, Stochastic differential equations for the non linear filtering problem, Osaka J. Math. 9, 19 (1972).

[47] H. Kunita and S. Watanabe, On square integrable martingales, Nagoya Math. J. 30, 209 (1967).

[48] O. Pohl, M. Hintsche, Z. Alirezaeizanjani, M. Seyrich, C. Beta, and H. Stark, Inferring the chemotactic strategy of p. putida and E. coli using modified kramersmoyal coefficients, PLoS Comput. Biol. 13, e1005329 (2017).

[49] S. Satoh, H. J. Kappen, and M. Saeki, An iterative method for nonlinear stochastic optimal control based on path integrals, IEEE Trans. Autom. Control 62, 262 (2016).

[50] P. Cluzel, M. Surette, and S. Leibler, An ultrasensitive bacterial motor revealed by monitoring signaling proteins in single cells, Science 287, 1652 (2000).

[51] Y. Tu, Quantitative modeling of bacterial chemotaxis: signal amplification and accurate adaptation, Annu. Rev. Biophys. 42, 337 (2013).

[52] J. M. Keegstra, K. Kamino, F. Anquez, M. D. Lazova, T. Emonet, and T. S. Shimizu, Phenotypic diversity and temporal variability in a bacterial signaling network revealed by singlecell FRET, Elife 6, e27455 (2017).

[53] R. Stocker, Reverse and flick: Hybrid locomotion in bacteria, Proc. Natl. Acad. Sci. USA 108, 2635 (2011).

[54] A. Gomez-Marin and M. Louis, Active sensation during orientation behavior in the Drosophila larva: More sense than luck, Curr. Opin. Neurobiol. 22, 208 (2012).

[55] Y. V. Kalinin, L. Jiang, Y. Tu, and M. Wu, Logarithmic sensing in Escherichia coli bacterial chemotaxis, Biophys. J. 96, 2439 (2009).

[56] Y. Yang, A. M. Pollard, C. H'ofler, G. Poschet, M. Wirtz, R. Hell, and V. Sourjik, Relation between chemotaxis and 
consumption of amino acids in bacteria, Mol. Microbiol. 96, 1272 (2015).

[57] X. Zhang, G. Si, Y. Dong, K. Chen, Q. Ouyang, C. Luo, and Y. Tu, Escape band in Escherichia coli chemotaxis in opposing attractant and nutrient gradients, Proc. Natl. Acad. Sci. USA 116, 2253 (2019).
[58] S. Rengarajan and E. A. Hallem, Olfactory circuits and behaviors of nematodes, Curr. Opin. Neurobiol. 41, 136 (2016).

[59] A. Tschantz, A. K. Seth, and C. L. Buckley, Learning actionoriented models through active inference, PLoS Comput. Biol. 16, 1 (2020). 